CrossMark \&click for updates

Cite this: Phys. Chem. Chem. Phys., $2016,18,32560$

Received 13th July 2016

Accepted 12th November 2016

DOI: $10.1039 / c 6 c p 04883 h$

www.rsc.org/pccp

\title{
Molecular electrometer and binding of cations to phospholipid bilayers $\dagger$
}

\author{
Andrea Catte, $\$^{\mathrm{a}}$ Mykhailo Girych, ${ }^{\mathrm{b}}$ Matti Javanainen, ${ }^{\mathrm{cd}}$ Claire Loison, ${ }^{\mathrm{e}}$ Josef Melcr, ${ }^{\mathrm{fg}}$ \\ Markus S. Miettinen, ${ }^{\text {hi } L u c a ~ M o n t i c e l l i, ~ J u k k a ~ M a ̈ a ̈ t t a ̈, ~}{ }^{k}$ Vasily S. Oganesyan, ${ }^{2}$ \\ O. H. Samuli Ollila, ${ }^{\star b}$ Joona Tynkkynen ${ }^{c}$ and Sergey Vilov ${ }^{\mathrm{e}}$
}

\begin{abstract}
Despite the vast amount of experimental and theoretical studies on the binding affinity of cations - especially the biologically relevant $\mathrm{Na}^{+}$and $\mathrm{Ca}^{2+}-$ for phospholipid bilayers, there is no consensus in the literature. Here we show that by interpreting changes in the choline headgroup order parameters according to the 'molecular electrometer' concept [Seelig et al., Biochemistry, 1987, 26, 7535], one can directly compare the ion binding affinities between simulations and experiments. Our findings strongly support the view that in contrast to $\mathrm{Ca}^{2+}$ and other multivalent ions, $\mathrm{Na}^{+}$and other monovalent ions (except $\mathrm{Li}^{+}$) do not specifically bind to phosphatidylcholine lipid bilayers at sub-molar concentrations. However, the $\mathrm{Na}^{+}$binding affinity was overestimated by several molecular dynamics simulation models, resulting in artificially positively charged bilayers and exaggerated structural effects in the lipid headgroups. While qualitatively correct headgroup order parameter response was observed with $\mathrm{Ca}^{2+}$ binding in all the tested models, no model had sufficient quantitative accuracy to interpret the $\mathrm{Ca}^{2+}$ :lipid stoichiometry or the induced atomistic resolution structural changes. All scientific contributions to this open collaboration work were made publicly, using nmrlipids.blogspot.fi as the main communication platform.
\end{abstract}

\section{Introduction}

Due to its high physiological importance - nerve cell signalling being the prime example - interaction of cations with phospholipid membranes has been widely studied via theory, simulations, and experiments. The relative ion binding affinities are generally agreed to follow the Hofmeister series, ${ }^{1-9}$ however, consensus on the quantitative affinities is currently lacking. Until 1990, the consensus (documented in two extensive reviews ${ }^{2,3}$ ) was

\footnotetext{
${ }^{a}$ School of Chemistry, University of East Anglia, Norwich, NR4 7TJ, UK

${ }^{b}$ Department of Neuroscience and Biomedical Engineering, Aalto University, Espoo, Finland. E-mail: samuli.ollila@aalto.fi

${ }^{c}$ Department of Physics, Tampere University of Technology, Tampere, Finland

${ }^{d}$ Department of Physics, University of Helsinki, Helsinki, Finland

${ }^{e}$ Univ Lyon, Université Claude Bernard Lyon 1, CNRS, Institut Lumiére Matiére, F-69622, LYON, France

${ }^{f}$ Institute of Organic Chemistry and Biochemistry, Czech Academy of Sciences, Flemingovo nám. 2, 16610 Prague 6, Czech Republic

${ }^{g}$ Charles University in Prague, Faculty of Mathematics and Physics, Ke Karlovu 3, 12116 Prague 2, Czech Republic

${ }^{h}$ Fachbereich Physik, Freie Universität Berlin, Berlin, Germany

${ }^{i}$ Max Planck Institute of Colloids and Interfaces, Department of Theory and Bio-Systems, Potsdam, Germany

${ }^{j}$ Institut de Biologie et Chimie des Protéines (IBCP), CNRS UMR 5086, Lyon, France

${ }^{k}$ Department of Chemistry, Aalto University, Espoo, Finland

$\dagger$ Electronic supplementary information (ESI) available: 8 figures, detailed technical discussion and simulation details. See DOI: 10.1039/c6cp04883h

\$ The authors are listed in alphabetical order.
}

that while multivalent cations interact significantly with phospholipid bilayers, for monovalent cations (with the exception of $\mathrm{Li}^{+}$) the interactions are weak. This conclusion has since been strengthened by further studies showing that bilayer properties remain unaltered upon the addition of sub-molar concentrations of monovalent salt. ${ }^{4,10,11}$ Since 2000, however, another view has emerged, suggesting much stronger interactions between phospholipids and monovalent cations, and strong $\mathrm{Na}^{+}$binding in particular. ${ }^{6-9,12-18}$

The pre-2000 view has the experimental support that (in contrast to the significant effects caused by any multivalent cations) sub-molar concentrations of $\mathrm{NaCl}$ have a negligible effect on phospholipid infrared spectra, ${ }^{4}$ area per molecule, ${ }^{10}$ dipole potential, ${ }^{19}$ lateral diffusion, ${ }^{11}$ and choline head group order parameters; ${ }^{20}$ in addition, the water sorption isotherm of a NaCl-phospholipid system is highly similar to that of a pure $\mathrm{NaCl}$ solution - indicating that the ion-lipid interaction is very weak. ${ }^{4}$

The post-2000 'strong binding' view rests on experimental and above all simulational findings. At sub-molar $\mathrm{NaCl}$ concentrations, the rotational and translational dynamics of membraneembedded fluorescent probes decreased, ${ }^{7,9,12}$ and atomic force microscopy (AFM) experiments showed changes in bilayer hardness; ${ }^{14-18}$ in atomistic molecular dynamics (MD) simulations, phospholipid bilayers consistently bound $\mathrm{Na}^{+}$, although the binding strength depended on the model used. ${ }^{12,13,21-26}$ 
Some observables have been interpreted in favour of both views. For example, as the effect of monovalent ions (except $\mathrm{Li}^{+}$) on the phase transition temperature is tiny (compared to the effect of multivalent ions), it was initially interpreted as an indication that only multivalent ions and $\mathrm{Li}^{+}$specifically bind to phospholipid bilayers; ${ }^{2}$ however, such a small effect in calorimetric measurements was later interpreted to indicate that also $\mathrm{Na}^{+}$binds. ${ }^{8,12}$ Similarly, the lack of significant positive electrophoretic mobility of phosphatidylcholine (PC) vesicles in the presence of $\mathrm{NaCl}$ (again in contrast to multivalent ions and $\mathrm{Li}^{+}$) suggested weak binding of $\mathrm{Na}^{+} ;^{1,8,14,15,27}$ however, these data were also explained by a countering effect of the $\mathrm{Cl}^{-}$ions. ${ }^{22,28}$ Furthermore, to reduce the area per lipid in scattering experiments, molar concentrations of $\mathrm{NaCl}$ were required, ${ }^{10}$ indicating weak ion-lipid interaction; in MD simulations, however, already orders of magnitude lower concentrations resulted in $\mathrm{Na}^{+}$binding and a clear reduction of area per lipid. ${ }^{12,23}$ Finally, lipid lateral diffusion was unaltered by $\mathrm{NaCl}$ in noninvasive NMR experiments ${ }^{11}$ however, as it was reduced upon $\mathrm{Na}^{+}$binding in simulations, the reduced lateral diffusion of fluorescent probes $^{7,9,12}$ has been interpreted to support the post-2000 'strong binding' view.

In this paper, we set out to solve the apparent contradictions between the pre-2000 and post-2000 views. To this end, we employ the 'molecular electrometer' concept, according to which the changes in the $\mathrm{C}-\mathrm{H}$ order parameters of the $\alpha$ and $\beta$ carbons in the phospholipid head group (see Fig. 1) can be used to measure the ion affinity for a PC lipid bilayer. ${ }^{20,29-32}$ As the order parameters can be accurately measured in experiments and directly compared to simulations, ${ }^{33}$ applying the molecular electrometer as a function of cation concentration allows the comparison of binding affinity between simulations and experiments. In addition to demonstrating the usefulness of this general concept, we show that the response of the $\alpha$ and $\beta$ order parameters to penetrating cations is qualitatively correct in $\mathrm{MD}$ simulations, but that in several models the affinity of $\mathrm{Na}^{+}$for PC bilayers is grossly overestimated. Moreover, we show that the accuracy of lipid- $\mathrm{Ca}^{2+}$ interactions in current models is not enough for atomistic resolution interpretation of NMR experiments.

This work was done as an Open Collaboration at nmrlipids. blogspot.fi; all the related files ${ }^{34}$ and almost all the simulation data (https://zenodo.org/collection/user-nmrlipids) are openly available.

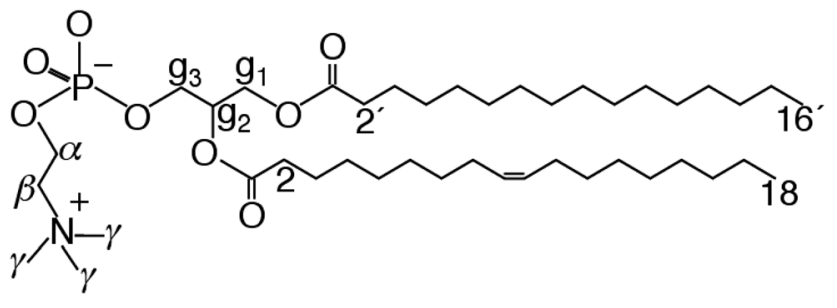

Fig. 1 Chemical structure of 1-palmitoyl-2-oleoylphosphatidylcholine (POPC), and the definition of $\gamma, \beta, \alpha, g_{1}, g_{2}$ and $g_{3}$ segments.

\section{Results and discussion}

\subsection{Background: molecular electrometer in experiments}

The basis for the molecular electrometer is the experimental observation that binding of any charged objects (ions, peptides, anesthetics, amphiphiles) on a PC bilayer interface induced systematic changes in the choline $\alpha$ and $\beta$ segment $\mathrm{C}-\mathrm{H}$ order parameters. $^{20,29-32,35-40}$ Being systematic, these changes could be employed for determining the binding affinities of the charged objects in question. Originally the molecular electrometer was devised for cations, ${ }^{20,29,30}$ but further experimental quantification with various positively and negatively charged molecules showed that the choline order parameters $S_{\mathrm{CH}}^{\alpha}$ and $S_{\mathrm{CH}}^{\beta}$ in general vary linearly with small amount of bound charge per lipid. ${ }^{30-32,35-40}$ Let now $S_{\mathrm{CH}}^{i}(0)$, where $i$ refers to either $\alpha$ or $\beta$, denote the order parameter in the absence of bound charge; the empirically observed linear relation can then be written as $^{41}$

$$
\Delta S_{\mathrm{CH}}^{i}=S_{\mathrm{CH}}^{i}\left(X^{ \pm}\right)-S_{\mathrm{CH}}^{i}(0)=\frac{4 m_{i}}{3 \chi} X^{ \pm} .
$$

Here $X^{ \pm}$is the amount of bound charge per lipid, $m_{i}$ an empirical constant depending on the valency and position of bound charge, and the value of the quadrupole coupling constant $\chi \approx 167 \mathrm{kHz}$.

With bound positive charge, the absolute value of the $\beta$ segment order parameter increases and the $\alpha$ segment order parameter decreases (and vice versa for negative charge). ${ }^{20,29-32,35,40}$ However, as $S_{\mathrm{CH}}^{\beta}(0)<0$ while $S_{\mathrm{CH}}^{\alpha}(0)>0,{ }^{42-44}$ both $\Delta S_{\mathrm{CH}}^{\beta}$ and $\Delta S_{\mathrm{CH}}^{\alpha}$ in fact decrease with bound positive charge (and increase with bound negative charge). Consequently, values of $m_{i}$ are negative for bound positive charges; for $\mathrm{Ca}^{2+}$ binding to POPC bilayer (in the presence of $100 \mathrm{mM} \mathrm{NaCl}$ ), combination of atomic absorption spectra and ${ }^{2} \mathrm{H}$ NMR experiments gave $m_{\alpha}=-20.5$ and $m_{\beta}=-10.0 .^{30}$ This decrease can be rationalised by electrostatically induced tilting of the choline $\mathrm{P}-\mathrm{N}$ dipole $\mathrm{e}^{31,32,46}-$ also seen in simulations ${ }^{23,24,47,48}$ - and is in line with the order parameter increase related to the $\mathrm{P}-\mathrm{N}$ vector tilting more parallel to the membrane plane seen with decreasing hydration levels. ${ }^{45}$

Quantification of $\Delta S_{\mathrm{CH}}^{\alpha}$ and $\Delta S_{\mathrm{CH}}^{\beta}$ for a wide range of different cations (aqueous cations, cationic peptides, cationic anesthetics) has revealed that $\Delta S_{\mathrm{CH}}^{\beta} / \Delta S_{\mathrm{CH}}^{\alpha} \approx 0.5 .^{38,40}$ More specifically, the relation $\Delta S_{\mathrm{CH}}^{\beta}=0.43 \Delta S_{\mathrm{CH}}^{\alpha}$ was found to hold for DPPC bilayers at various $\mathrm{CaCl}_{2}$ concentrations. ${ }^{20}$

\subsection{Molecular electrometer in MD simulations}

The black curves in Fig. 2 show how the headgroup order parameters for DPPC and POPC bilayers change in $\mathrm{H}^{2}$ NMR experiments as a function of salt solution concentration: ${ }^{20,30}$ Only minor changes are seen as a function of $[\mathrm{NaCl}]$, but the effect of $\left[\mathrm{CaCl}_{2}\right]$ is an order of magnitude larger. Thus, according to the molecular electrometer, the monovalent $\mathrm{Na}^{+}$ions have negligible affinity for PC lipid bilayers at concentrations up to $1 \mathrm{M}$, while binding of $\mathrm{Ca}^{2+}$ ions at the same concentration is significant. $^{20,30}$ 


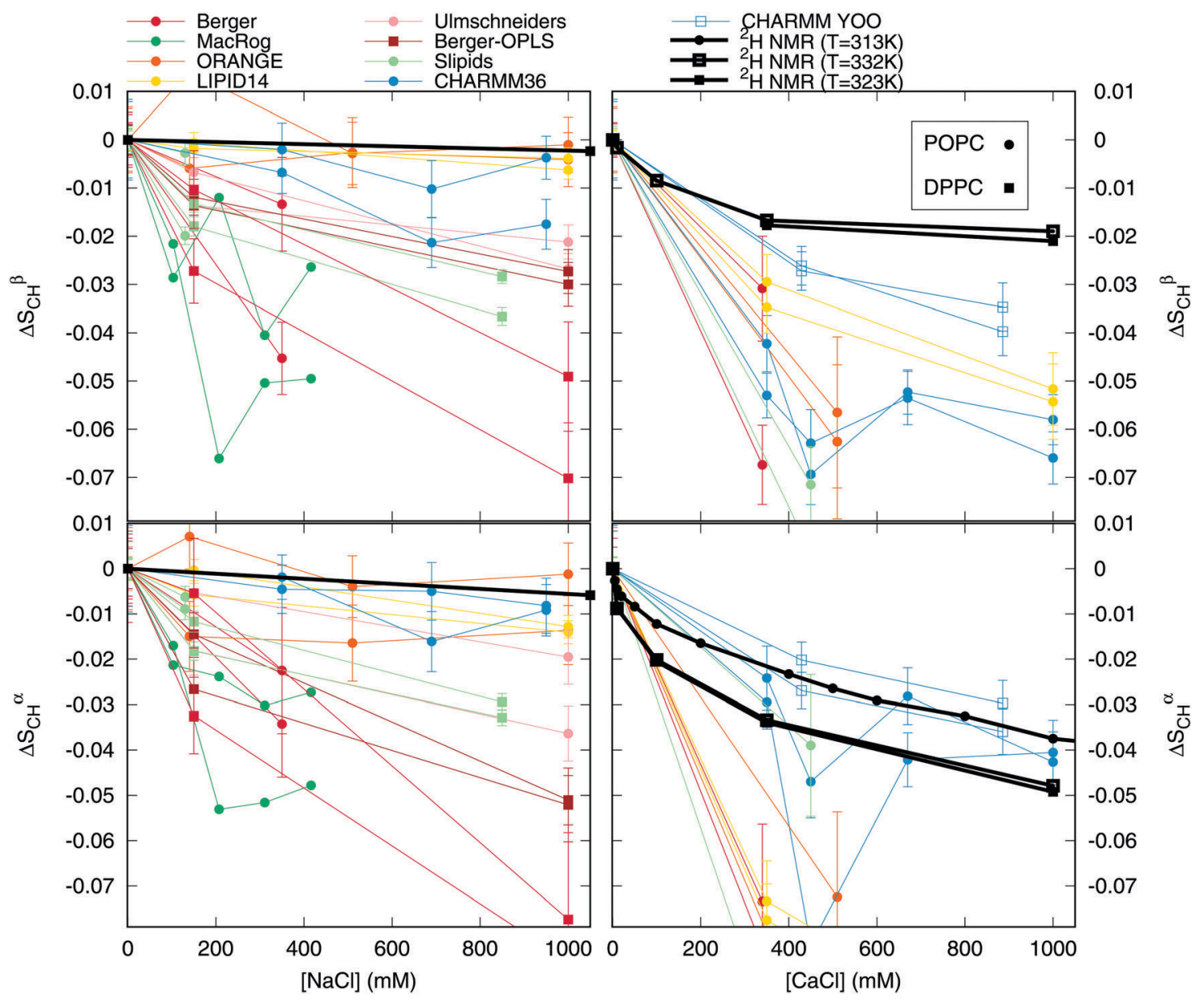

Fig. 2 Changes in the PC lipid headgroup $\beta$ (top row) and $\alpha$ (bottom) segment order parameters in response to $\mathrm{NaCl}_{\text {(left }}$ column) or $\mathrm{CaCl}_{2}$ (right column) salt solution concentration increase. Comparison between simulations (Table 1) and experiments (DPPCs from ref. 20, POPC from ref. 30). The signs of the experimental values, from experiments without ions, ${ }^{42-44}$ can be assumed unchanged at these salt concentrations. ${ }^{30,33}$ We stress that none of the models reproduces the order parameters without salt within experimental error, indicating structural inaccuracies of varying severity in all of them. ${ }^{45} \mathrm{Note}$ that the relatively large drop in CHARMM36 at $450 \mathrm{mM} \mathrm{CaCl} 2$ arose from more equilibrated binding due to a very long simulation time, see ESI. $\dagger$

Fig. 2 also reports order parameter changes calculated from MD simulations of DPPC and POPC lipid bilayers as a function of $\mathrm{NaCl}$ or $\mathrm{CaCl}_{2}$ initial concentrations in solution (for details of the simulated systems see Table 1 and ESI $\dagger$ ). Note that although none of these MD models reproduces within experimental uncertainty the order parameters for a pure PC bilayer without ions (Fig. 2 in ref. 45), which indicates structural inaccuracies of varying severity in all models,${ }^{45}$ all the models qualitatively reproduce the experimentally observed headgroup order parameter increase with dehydration. ${ }^{45}$ Similarly here (Fig. 2) the presence of cations led to the decrease of $S_{\mathrm{CH}}^{\alpha}$ and $S_{\mathrm{CH}}^{\beta}$, in qualitative agreement with experiments. The changes were, however, overestimated by most models, which according to the molecular electrometer indicates overbinding of cations in most MD simulations.

While the molecular electrometer is well established in experiments (see Section 2.1 above), it is not a priori clear that it works in simulations. The overestimated order parameter decrease could, in principle, arise from an exaggerated response of the choline headgroups to the binding cations, instead of overbinding.
Therefore, to evaluate the usability of the molecular electrometer in MD simulations, we analysed the relation between cation binding and choline order parameter decrease in simulations.

According to the molecular electrometer, the order parameter changes are linearly proportional to the amount of bound cations (eqn (1)). Fig. 3 shows this proportionality in MD simulations (see ESI $\dagger$ for the definition of bound ions); in keeping with the molecular electrometer, a roughly linear correlation between bound cation charge and order parameter change was found in all the eight models. Note that quantitative comparison of the proportionality constants (i.e. slopes in Fig. 3) between different models and experimental slopes $\left(m_{\alpha}=-20.5\right.$ and $m_{\beta}=-10.0$ for $\mathrm{Ca}^{2+}$ binding in DPPC bilayer in the presence of $100 \mathrm{mM} \mathrm{NaCl}^{30}$ ) is not straightforward since the simulation slopes depend on the definition used for bound ions (see ESI $\dagger$ ).

We note that the quantitative comparison of order parameter changes in response to bound charge should be more straightforward for systems with charged amphiphiles fully 
Table 1 List of MD simulations. The salt concentrations calculated as [salt] $=N_{\mathrm{c}} \times\left[\right.$ water] $/ N_{\mathrm{w}}$, where [water] $=55.5 \mathrm{M}$; these correspond to the concentrations reported in the experiments by Akutsu et al. ${ }^{20}$ The lipid force fields named as in our previous work ${ }^{45}$

\begin{tabular}{|c|c|c|c|c|c|c|c|c|c|c|}
\hline Force field for lipids/ions & Lipid & Salt & [Salt] (mM) & $N_{\mathrm{l}}^{a}$ & $N_{\mathrm{w}}{ }^{b}$ & $N_{\mathrm{c}}{ }^{c}$ & $T^{d}(\mathrm{~K})$ & $t_{\mathrm{sim}}^{e}(\mathrm{~ns})$ & $t_{\text {anal }}^{f}(\mathrm{~ns})$ & Files $^{g}$ \\
\hline Berger-POPC- $07^{51} /-$ & POPC & No & 0 & 128 & 7290 & 0 & 298 & 270 & 50 & 52 \\
\hline Berger-POPC- $07^{51} /$ ffgmx $^{53}$ & POPC & $\mathrm{NaCl}$ & 340 & 128 & 7202 & 44 & 298 & 110 & 50 & 54 \\
\hline Berger-POPC- $07^{51} /$ ffgmx $^{53}$ & POPC & $\mathrm{CaCl}_{2}$ & 340 & 128 & 7157 & 44 & 298 & 108 & 58 & 55 \\
\hline Berger-DPPC- $97^{56} /$ - & DPPC & No & 0 & 72 & 2880 & $\begin{array}{r}4+4 \\
0\end{array}$ & 323 & 60 & 50 & 57 \\
\hline Berger-DPPC- $97^{56} /$ ffgmx $^{53}$ & DPPC & $\mathrm{NaCl}$ & 150 & 72 & 2880 & 8 & 323 & 120 & 60 & 58 \\
\hline Berger-DPPC- $97^{56} /$ ffgmx $^{53}$ & DPPC & $\mathrm{NaCl}$ & 1000 & 72 & 2778 & 51 & 323 & 120 & 60 & 59 \\
\hline Berger-OPLS-DPPC- $06^{60} /-$ & DPPC & No & 0 & 72 & 2880 & 0 & 323 & 120 & 60 & 61 \\
\hline Berger-OPLS-DPPC- $06^{60} /$ OPLS $^{62}$ & DPPC & $\mathrm{NaCl}$ & 150 & 72 & 2880 & 8 & 323 & 120 & 60 & 63 \\
\hline Berger-OPLS-DPPC- $06^{60} /$ OPLS $^{62}$ & DPPC & $\mathrm{NaCl}$ & 1000 & 72 & 2778 & 51 & 323 & 120 & 60 & 64 \\
\hline CHARMM $36^{65} /-$ & POPC & No & 0 & 128 & 5210 & 0 & 303 & 200 & 150 & 66 \\
\hline CHARMM $36^{65} /-$ & POPC & No & 0 & 72 & 2242 & 0 & 303 & 30 & 20 & 67 \\
\hline CHARMM $36^{65} /$ CHARMM $36^{68}$ & POPC & $\mathrm{NaCl}$ & 350 & 72 & 2085 & 13 & 303 & 80 & 60 & 69 \\
\hline CHARMM $36^{65} /$ CHARMM $36^{68}$ & POPC & $\mathrm{NaCl}$ & 690 & 72 & 2085 & 26 & 303 & 73 & 60 & 70 \\
\hline CHARMM $36^{65} /$ CHARMM $36^{68}$ & POPC & $\mathrm{NaCl}$ & 950 & 72 & 2168 & 37 & 303 & 80 & 60 & 71 \\
\hline CHARMM $36^{65} /$ CHARMM 36 & POPC & $\mathrm{CaCl}_{2}$ & 350 & 128 & 6400 & 35 & 303 & 200 & 100 & 72 \\
\hline CHARMM $36^{65} /$ CHARMM36 & POPC & $\mathrm{CaCl}_{2}$ & 450 & 200 & 9000 & 73 & 310 & 2000 & 100 & 73 \\
\hline CHARMM $36^{65} /$ CHARMM 36 & POPC & $\mathrm{CaCl}_{2}$ & 670 & 128 & 6400 & 67 & 303 & 200 & 120 & 74 \\
\hline CHARMM $36^{65} /$ CHARMM 36 & POPC & $\mathrm{CaCl}_{2}$ & 1000 & 128 & 6400 & 100 & 303 & 200 & 100 & 75 \\
\hline CHARMM $36^{65} /-$ & DPPC & No & 0 & 128 & 8000 & 0 & 323 & 170 & 150 & - \\
\hline CHARMM $36^{65} / \mathrm{Yoo}^{76}$ & DPPC & $\mathrm{CaCl}_{2}$ & 430 & 128 & 7760 & 60 & 323 & 200 & 170 & - \\
\hline CHARMM $36^{65} / \mathrm{Yoo}^{76}$ & DPPC & $\mathrm{CaCl}_{2}$ & 890 & 128 & 7520 & 120 & 323 & 200 & 170 & - \\
\hline $\operatorname{MacRog}^{77} /$ - & POPC & No & 0 & 128 & 6400 & 0 & 310 & 400 & 200 & 78 \\
\hline MacRog $77 /$ - & POPC & No & 0 & 288 & 14400 & 0 & 310 & 90 & 40 & 79 \\
\hline MacRog $^{77} /$ OPLS $^{62}$ & POPC & $\mathrm{NaCl}$ & 100 & 288 & 14554 & 27 & 310 & 90 & 50 & 80 \\
\hline MacRog $^{77} / \mathrm{OPLS}^{62}$ & POPC & $\mathrm{NaCl}$ & 210 & 288 & 14500 & 54 & 310 & 90 & 50 & 80 \\
\hline MacRog $^{77} /$ OPLS $^{62}$ & POPC & $\mathrm{NaCl}$ & 310 & 288 & 14446 & 81 & 310 & 90 & 50 & 80 \\
\hline MacRog $^{77} /$ OPLS $^{62}$ & POPC & $\mathrm{NaCl}$ & 420 & 288 & 14392 & 108 & 310 & 90 & 50 & 80 \\
\hline Orange/- & POPC & No & 0 & 72 & 2880 & 0 & 298 & 60 & 50 & 81 \\
\hline Orange/OPLS ${ }^{62}$ & POPC & $\mathrm{NaCl}$ & 140 & 72 & 2866 & 7 & 298 & 120 & 60 & 82 \\
\hline Orange/OPLS ${ }^{62}$ & POPC & $\mathrm{NaCl}$ & 510 & 72 & 2802 & 26 & 298 & 120 & 100 & 83 \\
\hline Orange/OPLS ${ }^{62}$ & POPC & $\mathrm{NaCl}$ & 1000 & 72 & 2780 & 50 & 298 & 120 & 80 & 84 \\
\hline Orange/OPLS & POPC & $\mathrm{CaCl}_{2}$ & 510 & 72 & 2802 & 26 & 298 & 120 & 60 & 85 \\
\hline Slipids ${ }^{86} /-$ & POPC & No & 0 & 128 & 5120 & 0 & 310 & 200 & 150 & 87 \\
\hline Slipids ${ }^{86} /$ AMBER $^{88}$ & POPC & $\mathrm{NaCl}$ & 130 & 200 & 9000 & 21 & 310 & 105 & 100 & 89 \\
\hline Slipids $^{86} /$ AMBER $^{62}$ & POPC & $\mathrm{CaCl}_{2}$ & 450 & 200 & 9000 & 73 & 310 & 2000 & 100 & 90 \\
\hline Slipids ${ }^{91} /-$ & DPPC & No & 0 & 128 & 3840 & 0 & 323 & 150 & 100 & 92 \\
\hline Slipids $^{91} /$ AMBER $^{93,94}$ & DPPC & $\mathrm{NaCl}$ & 150 & 600 & 18000 & 49 & 323 & 100 & 40 & - \\
\hline Slipids ${ }^{91} /$ AMBER $^{93,94}$ & DPPC & $\mathrm{NaCl}$ & 850 & 128 & 3726 & 57 & 323 & 205 & 200 & 95 \\
\hline Slipids ${ }^{91} /$ AMBER $^{93,94}$ & DPPC & $\mathrm{NaCl}$ & 1750 & 128 & 3612 & 114 & 323 & 105 & 100 & 95 \\
\hline Slipids $^{91} /$ AMBER $^{93,94}$ & DPPC & $\mathrm{NaCl}$ & 2570 & 128 & 3514 & 163 & 323 & 105 & 100 & 95 \\
\hline Lipid14 ${ }^{96} / \ldots$ & POPC & No & 0 & 128 & 5120 & 0 & 298 & 205 & 200 & 97 \\
\hline Lipid14 ${ }^{96} / \mathrm{AMBER}^{62}$ & POPC & $\mathrm{NaCl}$ & 150 & 128 & 5120 & 12 & 298 & 205 & 200 & 98 \\
\hline Lipid $14^{96} /$ AMBER $^{62}$ & POPC & $\mathrm{NaCl}$ & 1000 & 128 & 5120 & 77 & 298 & 205 & 200 & 99 \\
\hline Lipid $14^{96} /$ AMBER $^{62}$ & POPC & $\mathrm{CaCl}_{2}$ & 350 & 128 & 6400 & 35 & 298 & 200 & 100 & 100 \\
\hline Lipid $14^{96} /$ AMBER $^{62}$ & POPC & $\mathrm{CaCl}_{2}$ & 1000 & 128 & 6400 & 100 & 298 & 200 & 100 & 101 \\
\hline Ulmschneiders ${ }^{102} /$ - & POPC & No & 0 & 128 & 5120 & 0 & 298 & $2 \times 205$ & $2 \times 200$ & 103 \\
\hline Ulmschneiders $^{102} / \mathrm{OPLS}^{62}$ & POPC & $\mathrm{NaCl}$ & 150 & 128 & 5120 & 12 & 298 & 205 & 200 & 104 \\
\hline Ulmschneiders $^{102} /$ OPLS $^{62}$ & POPC & $\mathrm{NaCl}$ & 1000 & 128 & 5120 & 77 & 298 & 205 & 200 & 105 \\
\hline
\end{tabular}

${ }^{a}$ Number of lipid molecules. ${ }^{b}$ Number of water molecules. ${ }^{c}$ Number of cations. ${ }^{d}$ Simulation temperature. ${ }^{e}$ Total simulation time. ${ }^{f}$ Time used for analysis. ${ }^{g}$ Reference for simulation files.

associated in the bilayer, as the amount of bound charge is then explicitly known in both simulations and experiments. In such a comparison between experiments ${ }^{32,49}$ and previously published Berger-model-based simulations, ${ }^{50}$ we could not rule out overestimation of order parameter response to bound cations (slopes $m_{\alpha}$ and $m_{\beta}$ ), see ESI. $\dagger$ This might, in principle, explain the overestimated order parameter response of the
Berger model to $\mathrm{CaCl}_{2}$, but not to $\mathrm{NaCl}$ (see discussion in $\mathrm{ESI} \dagger$ ). Since simulation data with charged amphiphiles are not available for other models, an extended comparison with different models is left for further studies.

Fig. 3 shows that the decrease of order parameters clearly correlated with the amount of bound cations in simulations. This is also evident from Fig. 4, which shows the $\mathrm{Na}^{+}$density 


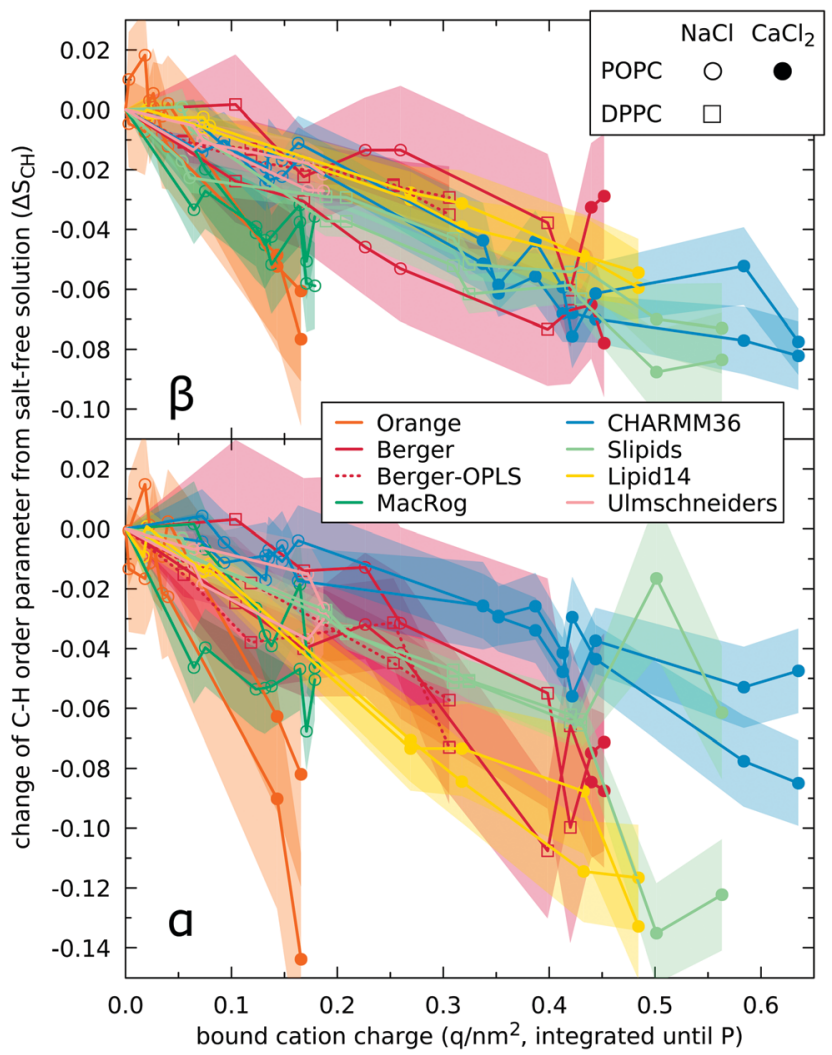

Fig. 3 Change of order parameters (from salt-free solution) of the $\beta$ and $\alpha$ segments, $\Delta S_{C H}^{\beta}$ and $\Delta S_{C H}^{\alpha}$, as a function of bound cation charge. Eight MD simulation models compared; the two lines per model denote to the two hydrogens per carbon. The order parameters as well as the bound charge calculated separately for each leaflet; cations residing between the bilayer centre and the density maximum of phosphorus considered bound; error bars (shaded) show standard error of mean over lipids.

profiles of the MD models ordered according to the order parameter change (in Fig. 2) from the smallest (top) to the largest (bottom). The general trend in the figure is that the $\mathrm{Na}^{+}$ density peaks are larger for models with larger changes in order parameters, in line with the observed correlation between cation binding and order parameter decrease in Fig. 3.

Fig. 5 compares the relation between $\Delta S_{\mathrm{CH}}^{\beta}$ and $\Delta S_{\mathrm{CH}}^{\alpha}$ in experiments ${ }^{20}$ and in MD models. Only Lipid14 gave $\Delta S_{\mathrm{CH}}^{\beta} / \Delta S_{\mathrm{CH}}^{\alpha}$ ratio in agreement with the experimental ratio; all other models underestimated the $\alpha$ segment order parameter decrease with bound cations with respect to the $\beta$ segment decrease.

In conclusion, a clear correlation between bound cations and order parameter decrease was observed for all simulation models. Consequently, the molecular electrometer can be used to compare the cation binding affinity between experiments and simulations. However, we found that quantitatively the response of $\alpha$ and $\beta$ segment order parameters to bound cations in simulations did not generally agree with the experiments; e.g., the $\Delta S_{\mathrm{CH}}^{\beta} / \Delta S_{\mathrm{CH}}^{\alpha}$ ratio agreed with experiments only in the Lipid14 model (Fig. 5). Thus, the observed overestimation of the order parameter changes with salt concentrations could, in principle, arise from overbinding of cations or from an oversensitive lipid headgroup response to the bound cations

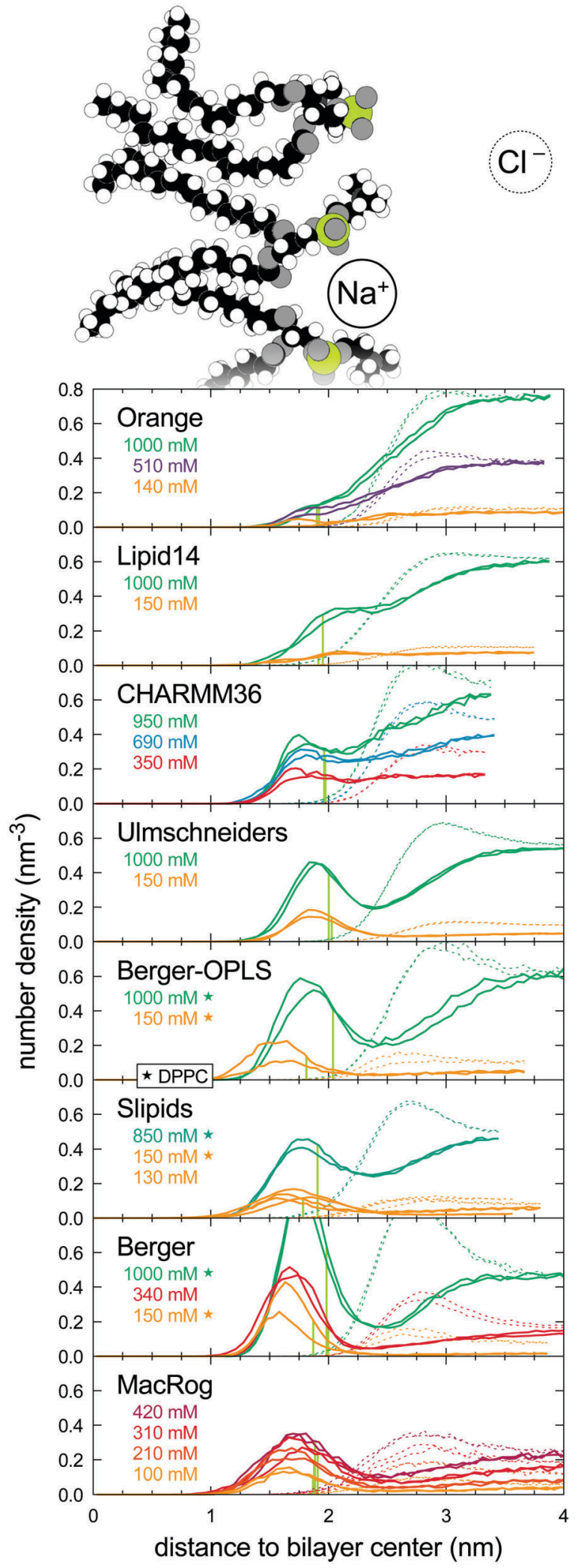

Fig. $4 \mathrm{Na}^{+}$(solid line) and $\mathrm{Cl}^{-}$(dashed) distributions along the lipid bilayer normal from MD simulations at several $\mathrm{NaCl}$ concentrations. The eight MD models are ordered according to their strength of order parameter change in response to $\mathrm{NaCl}$ (Fig. 2) from the weakest (top panel) to the strongest (bottom). The light green vertical lines indicate the locations of the phosphorus maxima, used to define bound cations in Fig. 3 . 


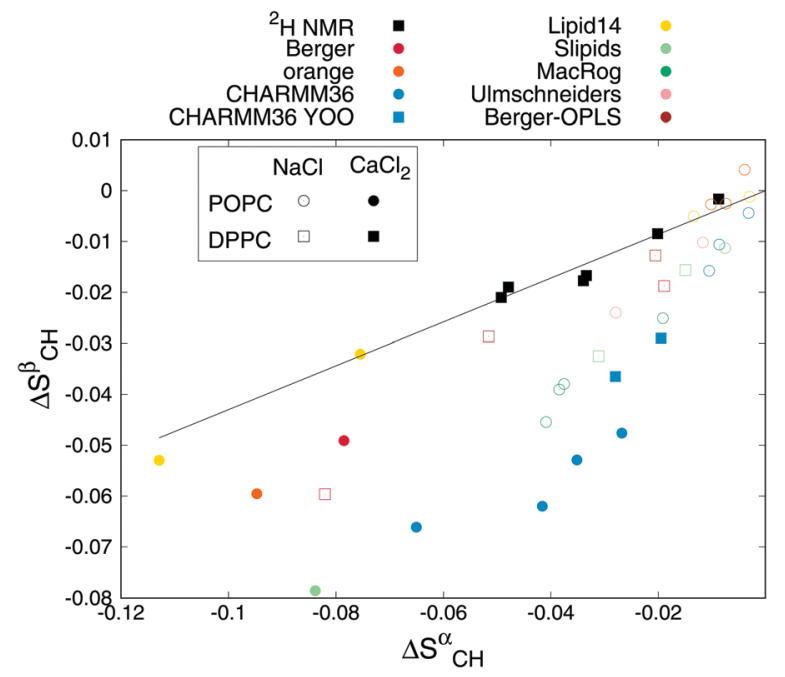

Fig. 5 Relation between $\Delta S_{C H}^{\beta}$ and $\Delta S_{C H}^{\alpha}$ from experiments ${ }^{20}$ and different simulation models. Solid line is $\Delta S_{C H}^{\beta}=0.43 \Delta S_{C H}^{\alpha}$ determined for DPPC bilayer from ${ }^{2} \mathrm{H}$ NMR experiment with various $\mathrm{CaCl}_{2}$ concentrations. ${ }^{20}$

(see also discussion in ESI $\dagger$ ). A careful analysis with current lipid models is performed in the next section.

\subsection{Cation binding in different simulation models}

The order parameter changes (Fig. 2) and density distributions (Fig. 4) demonstrated significantly different $\mathrm{Na}^{+}$binding affinities in different simulation models. The best agreement with experiments (lowest $\Delta S_{\mathrm{CH}}^{\alpha}$ and $\Delta S_{\mathrm{CH}}^{\beta}$ ) was observed for the three models (Orange, Lipid14, CHARMM36; see Fig. 2) that predicted the lowest $\mathrm{Na}^{+}$densities near the bilayer (Fig. 4). All the other models clearly overestimated the choline order parameter responses to $\mathrm{NaCl}$ (Fig. 2) - and notably the strength of the overestimation was clearly linked to the strength of the $\mathrm{Na}^{+}$binding affinity (compare Fig. 2 and 4), which leads us to conclude that $\mathrm{Na}^{+}$binding affinity was overestimated in all these models.

As in the best three models the order parameter changes with $\mathrm{NaCl}$ were small $(<0.02)$, the achieved statistical accuracy did not allow us to conclude which of the three had the most realistic $\mathrm{Na}^{+}$binding affinity, especially at physiological $\mathrm{NaCl}$ concentrations $(\sim 150 \mathrm{mM})$ relevant for most applications. The overestimated binding in the other models raises questions concerning the quality of predictions from these models when $\mathrm{NaCl}$ is present. Especially interactions between charged molecules and the bilayer might be significantly affected by the strong $\mathrm{Na}^{+}$binding, which gives the otherwise neutral bilayer an effective positive charge.

Significant $\mathrm{Ca}^{2+}$ binding affinity for phosphatidylcholine bilayers at sub-molar concentrations is agreed on in the literature, ${ }^{2,3,20,30}$ however, several details remain under discussion. Simulations suggest that $\mathrm{Ca}^{2+}$ binds to lipid carbonyl oxygens with a coordination number of $4.2,{ }^{13}$ while interpretation of NMR and scattering experiments suggest that one $\mathrm{Ca}^{2+}$ interacts mainly with the choline groups ${ }^{106-108}$ of two phospholipid molecules. ${ }^{30}$ A simulation model correctly reproducing the order parameter changes would resolve the discussion by giving atomistic resolution interpretation for the experiments.

As a function of $\mathrm{CaCl}_{2}$ concentration, all models but one (CHARMM36 with the recent heptahydrated $\mathrm{Ca}^{2+}$ by Yoo et $a .^{76}$ ) overestimated the order parameter decrease (Fig. 2), which according to the molecular electrometer indicates too strong $\mathrm{Ca}^{2+}$ binding. (We note that while this is the most likely scenario for the models that overestimated changes in both order parameters, for $\mathrm{CaCl}_{2}$ it is possible also that the headgroup response is oversensitive to bound cations, see ESI. $\dagger$ ) In CHARMM36 with the heptahydrated $\mathrm{Ca}^{2+}$ by Yoo et al., ${ }^{76}$ $\Delta S_{\mathrm{CH}}^{\beta}$ was overestimated but $\Delta S_{\mathrm{CH}}^{\alpha}$ underestimated (Fig. 2), in line with the $\Delta S_{\mathrm{CH}}^{\beta} / \Delta S_{\mathrm{CH}}^{\alpha}$ ratio in CHARMM36 being larger than in experiments (Fig. 5). As we do not know whether $\Delta S_{\mathrm{CH}}^{\beta}$ or $\Delta S_{\mathrm{CH}}^{\alpha}$ was more realistic, we cannot conclude whether $\mathrm{Ca}^{2+}$ binding was too strong or too weak in CHARMM36. This could be resolved by comparing against experimental data with a known amount of bound charge (e.g., amphiphilic cations ${ }^{32,49}$ ), however, such simulation data are not currently available.

The density distributions with $\mathrm{CaCl}_{2}$ showed significant $\mathrm{Ca}^{2+}$ binding in all models (Fig. 6), however, some differences occurred in details. The Berger model predicted deeper penetration (density maximum at $\sim 1.8 \mathrm{~nm}$ ) compared to other models $(\sim 2 \mathrm{~nm})$; the latter value is probably more realistic as ${ }^{1} \mathrm{H}$ NMR and neutron scattering data indicate that $\mathrm{Ca}^{2+}$ interacts mainly with the choline group. ${ }^{2,106-108}$ In CHARMM36 (but not in Slipids) practically all $\mathrm{Ca}^{2+}$ ions present in the simulation bound the bilayer within $2 \mu \mathrm{s}$ (Fig. 6 and ESI $\dagger$ ), which hints that the $\mathrm{Ca}^{2+}$ binding affinity of CHARMM36 is among the strongest of these models.

The origin of inaccuracies in lipid-ion interactions and binding affinities is far from clear. Potential candidates are, e.g., discrepancies in the ion models, ${ }^{109-111}$ incomplete treatment of electronic polarizability, ${ }^{112}$ and inaccuracies in the lipid headgroup description. ${ }^{45}$

Considering the ion models, Cordomi et al. ${ }^{24}$ showed the $\mathrm{Na}^{+}$binding affinity to decrease when ion radius is increased; however, in their DPPC bilayer simulations (with the OPLS-AA force field ${ }^{113}$ ) even the largest $\mathrm{Na}^{+}$radii still resulted in significant binding. In our results, the Slipids force field gave essentially similar binding affinity with ion parameters from ref. 88, 93 and 94 (Fig. 4). Further, compensation of missing electronic polarizability by scaling the ion charge ${ }^{112,114}$ reduced $\mathrm{Na}^{+}$binding in Berger, Berger-OPLS and Slipids, but not enough to reach agreement with experiments (ESI $\dagger$ ). The charge-scaled $\mathrm{Ca}^{2+}$ model ${ }^{115}$ slightly reduced binding in CHARMM36, but did not have significant influence in Slipids (ESI $\dagger$ ). The heptahydrated $\mathrm{Ca}^{2+}$ ions by Yoo et al. ${ }^{76}$ significantly reduced $\mathrm{Ca}^{2+}$ binding in CHARMM36 (Fig. 6), however, the model must be further analysed to fully interpret the results.

The lipid models may also have a significant influence on ion binding behaviour. For example, the same ion model and non-bonded parameters are used in Orange and Berger-OPLS, ${ }^{60}$ but while $\mathrm{Na}^{+}$ion binding affinity appeared realistic in Orange, it was significantly overestimated in Berger-OPLS (Fig. 4). However, realistic $\mathrm{Na}^{+}$binding does not automatically imply realistic 


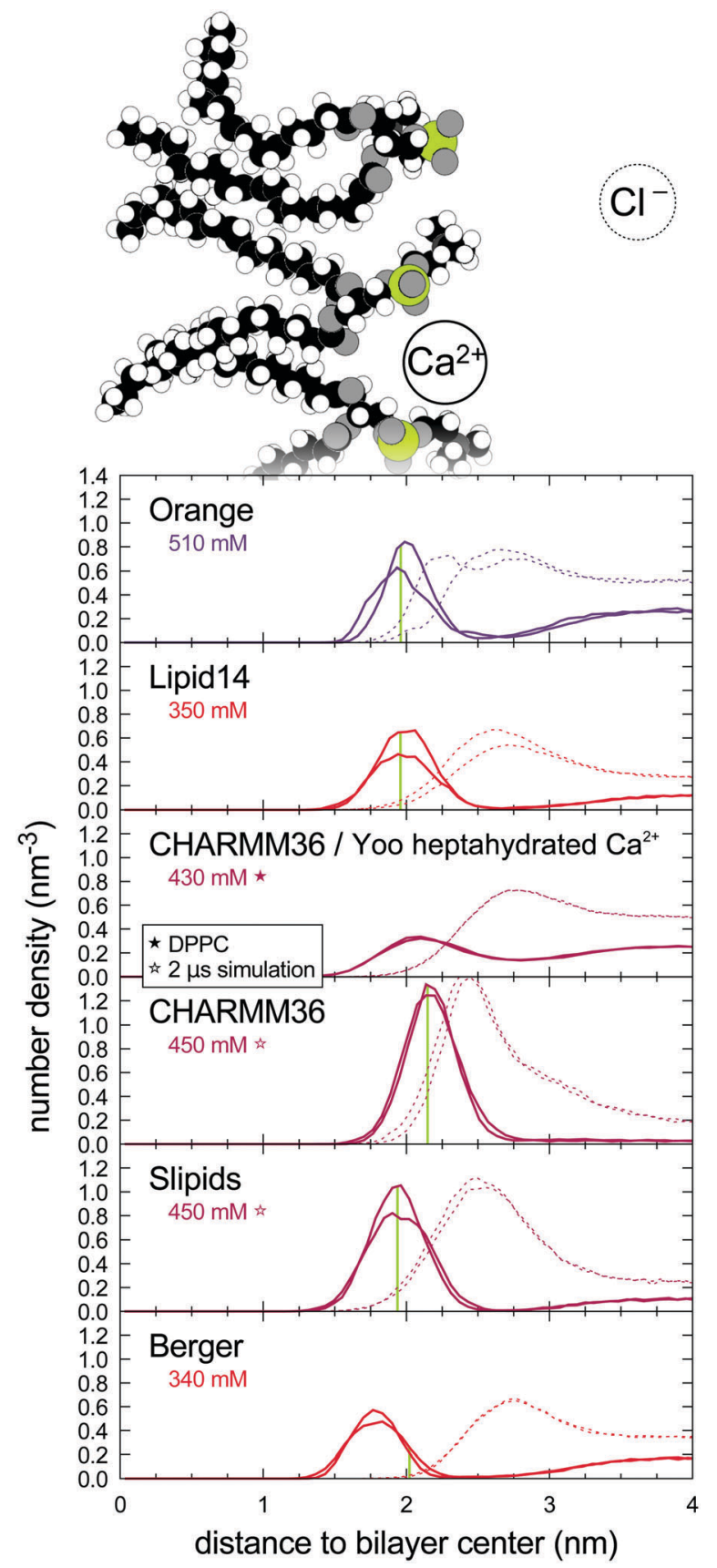

Fig. $6 \mathrm{Ca}^{2+}$ (solid line) and $\mathrm{Cl}^{-}$(dashed) distributions along the lipid bilayer normal from MD simulations. For clarity, only one $\mathrm{CaCl}_{2}$ concentration per MD model is shown; see ESI $\dagger$ for a plot including all the available concentrations. The light green vertical lines indicate the locations of the phosphorus maxima, used to define bound cations in Fig. 3.

$\mathrm{Ca}^{2+}$ binding (see Orange, Lipid14, and CHARMM36 in Fig. 2) or realistic choline order parameter response to bound charge (see Orange and CHARMM36 in Fig. 5). It should also be noted that the low binding affinity of $\mathrm{Na}^{+}$in CHARMM36 is due to the additional repulsion (NBFIX ${ }^{68}$ ) added between the sodium ions and lipid oxygens (ESI $\dagger$ ), and that in the $\mathrm{Ca}^{2+}$ model by Yoo et $a .^{76}$ the calcium is forced to be solvated solely by water. Altogether, our results indicate that probably both, lipid and ion force field parameters, need improvement to correctly predict the cation binding affinity, and the associated structural changes.

\section{Conclusions}

In accordance with the molecular electrometer, ${ }^{20,29-32}$ cation binding to lipid bilayers was accompanied with a decrease in the $\mathrm{C}-\mathrm{H}$ order parameters of the $\mathrm{PC}$ head group $\alpha$ and $\beta$ carbons in all the simulation models tested (Fig. 3) - despite of the known inaccuracies in the actual atomistic resolution structures. ${ }^{45}$ Hence, the molecular electrometer allowed a direct comparison of $\mathrm{Na}^{+}$binding affinity between simulations and noninvasive NMR experiments. The comparison revealed that most models overestimated $\mathrm{Na}^{+}$binding; only Orange, Lipid14, and CHARMM36 predicted realistic binding affinities. None of the tested models had the accuracy required to interpret the $\mathrm{Ca}^{2+}$ :lipid stoichiometry or the induced structural changes with atomistic resolution.

Taken together, our results corroborate the pre-2000 view that at sub-molar concentrations, in contrast to $\mathrm{Ca}^{2+}$ and other multivalent ions, ${ }^{1-4,10,11,19,20,27,30} \mathrm{Na}^{+}$and other monovalent ions (except $\mathrm{Li}^{+}$) do not specifically bind to phospholipid bilayers. Concerning the interpretation of existing experimental data, our work supports Cevc's view ${ }^{2}$ that the observed small shift in phase transition temperature is not indicative of $\mathrm{Na}^{+}$ binding. Further, our findings are in line with the noninvasive NMR spectroscopy work of Filippov et al. ${ }^{11}$ that proved the results of ref. 7, 9 and 12 to be explainable by direct interactions between $\mathrm{Na}^{+}$ions and fluorescent probes. Finally, as spectroscopic methods are in general more sensitive to atomistic details in fluid-like environment than AFM, our work indirectly suggests that the ion binding reported from AFM experiments on fluid-like lipid bilayer systems ${ }^{14-18}$ might be confounded with other physical features of the system. Concerning contradictions in MD simulation results, we reinterpret the strong $\mathrm{Na}^{+}$binding as an artefact of several simulation models, e.g., the Berger model used in ref. 12 and 13.

The artificial specific $\mathrm{Na}^{+}$binding in MD simulations may lead to doubtful results, as it effectively results in a positively charged phosphatidylcholine lipid bilayer even at physiological $\mathrm{NaCl}$ concentrations. Such a charged bilayer will have distinctly different interactions with charged objects than what a (more realistic) model without specific $\mathrm{Na}^{+}$binding would predict. Furthermore, the overestimation of binding affinity may extend from ions to other positively charged objects, say, membrane protein segments. This would affect lipid-protein interactions and could explain, for example, certain contradicting results on electrostatic interactions between charged protein segments and lipid bilayers. ${ }^{116,117}$ In conclusion, more careful studies and model development on lipid bilayer-charged object interactions are urgently called for to make molecular dynamics simulations directly usable in a physiologically relevant electrolytic environment.

This work was done as a fully open collaboration, using nmrlipids.blogspot.fi as the communication platform. All the 
scientific contributions were communicated publicly through this blog or the GitHub repository. ${ }^{34}$ All the related content and data are available at ref. 34 .

\section{Acknowledgements}

AC and VSO wish to thank the Research Computing Service at UEA for access to the High Performance Computing Cluster; VSO acknowledges the Engineering and Physical Sciences Research Council in the UK for financial support (EP/L001322/1). MG acknowledges financial support from Finnish Center of International Mobility (Fellowship TM-9363). J. Melcr acknowledges computational resources provided by the CESNET LM2015042 and the CERIT Scientific Cloud LM2015085 projects under the program "Projects of Large Research, Development, and Innovations Infrastructure". MSM acknowledges financial support from the Volkswagen Foundation (86110). LM acknowledges funding from the Institut National de la Sante et de la Recherche Medicale (INSERM). OHSO acknowledges Tiago Ferreira for very useful discussions, the Emil Aaltonen foundation for financial support, Aalto Science-IT project and CSC-IT Center for Science for computational resources.

\section{References}

1 M. Eisenberg, T. Gresalfi, T. Riccio and S. McLaughlin, Biochemistry, 1979, 18, 5213-5223.

2 G. Cevc, Biochim. Biophys. Acta, Rev. Biomembr., 1990, 1031, 311-382.

3 J.-F. Tocanne and J. Teissié, Biochim. Biophys. Acta, Rev. Biomembr., 1990, 1031, 111-142.

4 H. Binder and O. Zschörnig, Chem. Phys. Lipids, 2002, 115, 39-61.

5 J. J. Garcia-Celma, L. Hatahet, W. Kunz and K. Fendler, Langmuir, 2007, 23, 10074-10080.

6 E. Leontidis and A. Aroti, J. Phys. Chem. B, 2009, 113, 1460-1467.

7 R. Vacha, S. W. I. Siu, M. Petrov, R. A. Böckmann, J. BaruchaKraszewska, P. Jurkiewicz, M. Hof, M. L. Berkowitz and P. Jungwirth, J. Phys. Chem. A, 2009, 113, 7235-7243.

8 B. Klasczyk, V. Knecht, R. Lipowsky and R. Dimova, Langmuir, 2010, 26, 18951-18958.

9 F. F. Harb and B. Tinland, Langmuir, 2013, 29, 5540-5546.

10 G. Pabst, A. Hodzic, J. Strancar, S. Danner, M. Rappolt and P. Laggner, Biophys. J., 2007, 93, 2688-2696.

11 A. Filippov, G. Orädd and G. Lindblom, Chem. Phys. Lipids, 2009, 159, 81-87.

12 R. A. Böckmann, A. Hac, T. Heimburg and H. Grubmüller, Biophys. J., 2003, 85, 1647-1655.

13 R. A. Böckmann and H. Grubmüller, Angew. Chem., Int. Ed., 2004, 43, 1021-1024.

14 S. Garcia-Manyes, G. Oncins and F. Sanz, Biophys. J., 2005, 89, 1812-1826.

15 S. Garcia-Manyes, G. Oncins and F. Sanz, Electrochim. Acta, 2006, 51, 5029-5036.
16 T. Fukuma, M. J. Higgins and S. P. Jarvis, Phys. Rev. Lett., 2007, 98, 106101.

17 U. Ferber, G. Kaggwa and S. Jarvis, Eur. Biophys. J., 2011, 40, 329-338.

18 L. Redondo-Morata, G. Oncins and F. Sanz, Biophys. J., 2012, 102, 66-74.

19 R. J. Clarke and C. Lüpfert, Biophys. J., 1999, 76, 2614-2624.

$20 \mathrm{H}$. Akutsu and J. Seelig, Biochemistry, 1981, 20, 7366-7373.

21 J. N. Sachs, H. Nanda, H. I. Petrache and T. B. Woolf, Biophys. J., 2004, 86, 3772-3782.

22 M. L. Berkowitz, D. L. Bostick and S. Pandit, Chem. Rev., 2006, 106, 1527-1539.

23 A. Cordomí, O. Edholm and J. J. Perez, J. Phys. Chem. B, 2008, 112, 1397-1408.

24 A. Cordomí, O. Edholm and J. J. Perez, J. Chem. Theory Comput., 2009, 5, 2125-2134.

25 C. Valley, J. Perlmutter, A. Braun and J. Sachs, J. Membr. Biol., 2011, 244, 35-42.

26 M. L. Berkowitz and R. Vacha, Acc. Chem. Res., 2012, 45, 74-82.

27 S. A. Tatulian, Eur. J. Biochem., 1987, 170, 413-420.

28 V. Knecht and B. Klasczyk, Biophys. J., 2013, 104, 818-824.

29 M. F. Brown and J. Seelig, Nature, 1977, 269, 721-723.

30 C. Altenbach and J. Seelig, Biochemistry, 1984, 23, 3913-3920.

31 J. Seelig, P. M. MacDonald and P. G. Scherer, Biochemistry, 1987, 26, 7535-7541.

32 P. G. Scherer and J. Seelig, Biochemistry, 1989, 28, 7720-7728.

33 O. H. S. Ollila and G. Pabst, Biochim. Biophys. Acta, 2016, 1858, 2512-2528.

34 A. Catte, M. Girych, M. Javanainen, C. Loison, J. Melcr, M. S. Miettinen, L. Monticelli, J. Määttä, V. S. Oganesyan, O. H. S. Ollila, J. Tynkkynen and S. Vilov, NMRLipids/ lipid_ionINTERACTION: Final submission to Physical Chemistry Chemical Physics, 2016, DOI: 10.5281/zenodo.167370.

35 C. Altenbach and J. Seelig, Biochim. Biophys. Acta, 1985, 818, 410-415.

36 P. M. Macdonald and J. Seelig, Biochemistry, 1987, 26, 1231-1240.

37 M. Roux and M. Bloom, Biochemistry, 1990, 29, 7077-7089.

38 G. Beschiaschvili and J. Seelig, Biochim. Biophys. Acta, Biomembr., 1991, 1061, 78-84.

39 F. M. Marassi and P. M. Macdonald, Biochemistry, 1992, 31, 10031-10036.

40 J. R. Rydall and P. M. Macdonald, Biochemistry, 1992, 31, 1092-1099.

41 T. M. Ferreira, R. Sood, R. Bärenwald, G. Carlström, D. Topgaard, K. Saalwächter, P. K. J. Kinnunen and O. H. S. Ollila, Langmuir, 2016, 32, 6524-6533.

42 M. Hong, K. Schmidt-Rohr and A. Pines, J. Am. Chem. Soc., 1995, 117, 3310-3311.

43 M. Hong, K. Schmidt-Rohr and D. Nanz, Biophys. J., 1995, 69, 1939-1950.

44 J. D. Gross, D. E. Warschawski and R. G. Griffin, J. Am. Chem. Soc., 1997, 119, 796-802.

45 A. Botan, F. Favela-Rosales, P. F. J. Fuchs, M. Javanainen, M. Kanduč, W. Kulig, A. Lamberg, C. Loison, A. Lyubartsev, 
M. S. Miettinen, L. Monticelli, J. Määttä, O. H. S. Ollila, M. Retegan, T. Róg, H. Santuz and J. Tynkkynen, J. Phys. Chem. B, 2015, 119, 15075-15088.

46 J. Seelig, Cell Biol. Int. Rep., 1990, 14, 353-360.

47 A. A. Gurtovenko, M. Miettinen, M. Karttunen and I. Vattulainen, J. Phys. Chem. B, 2005, 109, 21126-21134.

48 W. Zhao, A. A. Gurtovenko, I. Vattulainen and M. Karttunen, J. Phys. Chem. B, 2012, 116, 269-276.

49 C. M. Franzin, P. M. Macdonald, A. Polozova and F. M. Winnik, Biochim. Biophys. Acta, Biomembr., 1998, 1415, 219-234.

50 M. S. Miettinen, A. A. Gurtovenko, I. Vattulainen and M. Karttunen, J. Phys. Chem. B, 2009, 113, 9226-9234.

51 S. Ollila, M. T. Hyvönen and I. Vattulainen, J. Phys. Chem. B, 2007, 111, 3139-3150.

52 O. H. S. Ollila, T. Ferreira and D. Topgaard, MD simulation trajectory and related files for POPC bilayer (Berger model delivered by Tieleman, Gromacs 4.5), 2014, DOI: 10.5281/ zenodo.13279.

53 T. P. Straatsma and H. J. C. Berendsen, J. Chem. Phys., 1988, 89, 5876-5886.

54 O. H. S. Ollila, MD simulation trajectory and related files for POPC bilayer with $340 \mathrm{mM} \mathrm{NaCl}$ (Berger model delivered by Tieleman, ffgmx ions, Gromacs 4.5), 2015, DOI: 10.5281/ zenodo.32144.

55 O. H. S. Ollila, MD simulation trajectory and related files for POPC bilayer with $340 \mathrm{mM} \mathrm{CaCl \_ 2} \mathrm{(Berger} \mathrm{model} \mathrm{delivered} \mathrm{by}$ Tieleman, ffgmx ions, Gromacs 4.5), 2015, DOI: 10.5281/ zenodo.32173.

56 S.-J. Marrink, O. Berger, P. Tieleman and F. Jähnig, Biophys. J., 1998, 74, 931-943.

57 J. Määttä, DPPC_Berger, 2015, DOI: 10.5281/zenodo.13934.

58 J. Määttä, DPPC_Berger_NaCl, 2015, DOI: 10.5281/zenodo. 16319.

59 J. Määttä, DPPC_Berger_NaCl_1Mol, 2015, DOI: 10.5281/ zenodo.17210.

60 D. P. Tieleman, J. L. MacCallum, W. L. Ash, C. Kandt, Z. Xu and L. Monticelli, J. Phys.: Condens. Matter, 2006, 18, S1221.

61 J. Määttä, DPPC_Berger_OPLSO6, 2015, DOI: 10.5281/zenodo. 17237.

62 J. Åqvist, J. Phys. Chem., 1990, 94, 8021-8024.

63 J. Määttä, DPPC_Berger_OPLSO6_NaCl, 2015, DOI: 10.5281/ zenodo.16484.

64 J. Määttä, DPPC_Berger_OPLSO6_NaCl_1Mol, 2016, DOI: 10.5281/zenodo.46152.

65 J. B. Klauda, R. M. Venable, J. A. Freites, J. W. O’Connor, D. J. Tobias, C. Mondragon-Ramirez, I. Vorobyov, A. D. Mackerell Jr. and R. W. Pastor, J. Phys. Chem. B, 2010, 114, 7830-7843.

$66 \mathrm{H}$. Santuz, MD simulation trajectory and related files for POPC bilayer (CHARMM36, Gromacs 4.5), 2015, DOI: 10.5281/ zenodo.14066.

67 O. H. S. Ollila and M. Miettinen, MD simulation trajectory and related files for POPC bilayer (CHARMM36, Gromacs 4.5), 2015, DOI: 10.5281/zenodo.13944.

68 R. M. Venable, Y. Luo, K. Gawrisch, B. Roux and R. W. Pastor, J. Phys. Chem. B, 2013, 117, 10183-10192.
69 O. H. S. Ollila, MD simulation trajectory and related files for POPC bilayer with $350 \mathrm{mM} \mathrm{NaCl}$ (CHARMM36, Gromacs 4.5), 2015, DOI: 10.5281/zenodo.32496.

70 O. H. S. Ollila, MD simulation trajectory and related files for POPC bilayer with $690 \mathrm{mM} \mathrm{NaCl}$ (CHARMM36, Gromacs 4.5), 2015, DOI: 10.5281/zenodo.32497.

71 O. H. S. Ollila, MD simulation trajectory and related files for POPC bilayer with $950 \mathrm{mM} \mathrm{NaCl}$ (CHARMM36, Gromacs 4.5), 2015, DOI: 10.5281/zenodo.32498.

72 M. Girych and O. H. S. Ollila, POPC_CHARMM36_CaCl2_035Mol, 2015, DOI: 10.5281/zenodo.35159.

73 M. Javanainen, POPC@310K, 450 mM of CaCl_2. Charmm36 with default Charmm ions, 2016, DOI: 10.5281/zenodo. 51185.

74 M. Girych and O. H. S. Ollila, POPC_CHARMM36_CaCl2_ 067Mol, 2015, DOI: 10.5281/zenodo.35160.

75 M. Girych and O. H. S. Ollila, POPC_CHARMM36_CaCl2_ 1Mol, 2015, DOI: 10.5281/zenodo.35156.

76 J. Yoo, J. Wilson and A. Aksimentiev, Biopolymers, 2016, 105, 752-763.

77 A. Maciejewski, M. Pasenkiewicz-Gierula, O. Cramariuc, I. Vattulainen and T. Rog, J. Phys. Chem. B, 2014, 118, 4571-4581.

78 M. Javanainen and W. Kulig, POPC/Cholesterol@310K. 0, 10, 40, 50 and 60 mol-\% cholesterol. Model by Maciejewski and Rog, 2015, DOI: 10.5281/zenodo.13877.

79 M. Javanainen, POPC@310K, varying water-to-lipid ratio, Model by Maciejewski and Rog, 2014, DOI: 10.5281/zenodo. 13498.

80 M. Javanainen and J. Tynkkynen, POPC@310K, varying amounts of NaCl. Model by Maciejewski and Rog, 2015, DOI: 10.5281/zenodo.14976.

81 O. H. S. Ollila, J. Määttä and L. Monticelli, MD simulation trajectory for POPC bilayer (Orange, Gromacs 4.5.), 2015, DOI: $10.5281 /$ zenodo.34488.

82 O. H. S. Ollila, J. Määttä and L. Monticelli, MD simulation trajectory for POPC bilayer with $140 \mathrm{mM}$ NaCl (Orange, Gromacs 4.5.), 2015, DOI: 10.5281/zenodo.34491.

83 O. H. S. Ollila, J. Määttä and L. Monticelli, MD simulation trajectory for POPC bilayer with $510 \mathrm{mM} \mathrm{NaCl}$ (Orange, Gromacs 4.5.), 2015, DOI: 10.5281/zenodo.34490.

84 S. Ollila, J. Määttä and L. Monticelli, MD simulation trajectory for POPC bilayer with $1000 \mathrm{mM} \mathrm{NaCl}$ (Orange, Gromacs 4.5.), 2015, DOI: 10.5281/zenodo.34497.

85 O. H. S. Ollila, J. Määttä and L. Monticelli, MD simulation trajectory for POPC bilayer with $510 \mathrm{mM}$ CaCl_2 (Orange, Gromacs 4.5.), 2015, DOI: 10.5281/zenodo.34498.

86 J. P. M. Jämbeck and A. P. Lyubartsev, J. Chem. Theory Comput., 2012, 8, 2938-2948.

87 M. Javanainen, POPC@310K, Slipids force field., 2015, DOI: 10.5281/zenodo.13887.

88 D. E. Smith and L. X. Dang, J. Chem. Phys., 1994, 100, 3757-3766. 89 M. Javanainen, POPC@310K, $130 \mathrm{mM}$ of NaCl. Slipids with ions by Smith \& Dang, 2015, DOI: 10.5281/zenodo.35275.

90 M. Javanainen, POPC@310K, 450 mM of CaCl_2. Slipids with default Amber ions, 2016, DOI: 10.5281/zenodo.51182. 
91 J. P. M. Jämbeck and A. P. Lyubartsev, J. Phys. Chem. B, 2012, 116, 3164-3179.

92 J. Määttä, DPPC_Slipids, 2014, DOI: 10.5281/zenodo.13287. 93 D. Beglov and B. Roux, J. Chem. Phys., 1994, 100, 9050-9063. 94 B. Roux, Biophys. J., 1996, 71, 3177-3185.

$95 \mathrm{~J}$. Melcr, Simulation files for DPPC lipid membrane with Slipids force field for Gromacs MD simulation engine, 2016, DOI: 10.5281 /zenodo.55322.

96 C. J. Dickson, B. D. Madej, G. A. Skjevik, R. M. Betz, K. Teigen, I. R. Gould and R. C. Walker, J. Chem. Theory Comput., 2014, 10, 865-879.

97 M. Girych and O. H. S. Ollila, POPC_AMBER_LIPID14_ Verlet, 2015, DOI: 10.5281/zenodo.30898.

98 M. Girych and O. H. S. Ollila, POPC_AMBER_LIPID14_ NaCl_015Mol, 2015, DOI: 10.5281/zenodo.30891.

99 M. Girych and O. H. S. Ollila, POPC_AMBER_LIPID14_ NaCl_1Mol, 2015, DOI: 10.5281/zenodo.30865.

100 M. Girych and O. H. S. Ollila, POPC_AMBER_LIPID14_ CaCl2_035Mol, 2015, DOI: 10.5281/zenodo.34415.

$101 \mathrm{M}$. Girych and O. H. S. Ollila, POPC_AMBER_LIPID14_ CaCl2_1Mol, 2015, DOI: 10.5281/zenodo.35074.

102 J. P. Ulmschneider and M. B. Ulmschneider, J. Chem. Theory Comput., 2009, 5, 1803-1813.

103 M. Girych and O. H. S. Ollila, POPC_Ulmschneider_OPLS_ Verlet_Group, 2015, DOI: 10.5281/zenodo.30904.

104 M. Girych and O. H. S. Ollila, POPC_Ulmschneider_OPLS_ NaCl_015Mol, 2015, DOI: 10.5281/zenodo.30892.
105 M. Girych and O. H. S. Ollila, POPC_Ulmschneider_OPLS_ NaCl_1Mol, 2015, DOI: 10.5281/zenodo.30894.

106 H. Hauser, M. C. Phillips, B. Levine and R. Williams, Nature, 1976, 261, 390-394.

107 H. Hauser, W. Guyer, B. Levine, P. Skrabal and R. Williams, Biochim. Biophys. Acta, Biomembr., 1978, 508, 450-463.

108 L. Herbette, C. Napolitano and R. McDaniel, Biophys. J., 1984, 46, 677-685.

109 B. Hess, C. Holm and N. van der Vegt, J. Chem. Phys., 2006, 124, 164509.

110 A. A. Chen and R. V. Pappu, J. Phys. Chem. B, 2007, 111, 11884-11887.

111 M. M. Reif, M. Winger and C. Oostenbrink, J. Chem. Theory Comput., 2013, 9, 1247-1264.

112 I. Leontyev and A. Stuchebrukhov, Phys. Chem. Chem. Phys., 2011, 13, 2613-2626.

113 W. L. Jorgensen, D. S. Maxwell and J. Tirado-Rives, J. Am. Chem. Soc., 1996, 118, 11225-11236.

114 M. Kohagen, P. E. Mason and P. Jungwirth, J. Phys. Chem. $B, 2016,120,1454-1460$.

115 M. Kohagen, P. E. Mason and P. Jungwirth, J. Phys. Chem. $B, 2014$, 118, 7902-7909.

116 A. Arkhipov, Y. Shan, R. Das, N. Endres, M. Eastwood, D. Wemmer, J. Kuriyan and D. Shaw, Cell, 2013, 152, 557-569.

117 K. Kaszuba, M. Grzybek, A. Orlowski, R. Danne, T. Róg, K. Simons, Ü. Coskun and I. Vattulainen, Proc. Natl. Acad. Sci. U. S. A., 2015, 112, 4334-4339. 